\title{
Large-Eddy Simulation for Flow and Dispersion in Urban Streets
}

\author{
Zheng-Tong Xie ${ }^{1}$ \& Ian P Castro \\ School of Engineering Sciences, University of Southampton, SO17 1BJ, UK
}

\begin{abstract}
Large-eddy simulations (LES) with our recently developed inflow approach (Xie \& Castro, 2008a) have been used for flow and dispersion within a genuine city area the DAPPLE site, located at the intersection of Marylebone Rd and Gloucester Pl in Central London. Numerical results up to second-order statistics are reported for a computational domain of $1.2 \mathrm{~km}$ (streamwise) $\times 0.8 \mathrm{~km}$ (lateral) $\times 0.2 \mathrm{~km}$ (in full scale), with a resolution down to approximately one meter in space and one second in time. They are in reasonable agreement with the experimental data. Such a comprehensive urban geometry is often, as here, composed of staggered, aligned, square arrays of blocks with non-uniform height and non-uniform base, street canyons and intersections. Both the integrative and local effect of flow and dispersion to these geometrical patterns were investigated. For example, it was found that the peaks of spatially averaged $u_{r m s}, v_{r m s}, w_{r m s}$ and $\left\langle u^{\prime} w^{\prime}\right\rangle$ occurred neither at the mean height nor at the maximum height, but at the height of large and tall buildings. It was also found that the mean and fluctuating concentrations in the near-source field is highly dependent on the source location and the local geometry pattern, whereas in the far field (e.g. $>0.1 \mathrm{~km}$ ) they are not. In summary, it is demonstrated that full-scale resolution of around one meter is sufficient to yield accurate prediction of the flow and mean dispersion characteristics and to provide reasonable estimation of concentration fluctuations.
\end{abstract}

Key words: street scale flow, street scale dispersion, DAPPLE, wind direction, multiple-tracers

\section{INTRODUCTION}

Understanding the mechanisms of turbulence, dispersion and heat transfer in urban streets is becoming increasedly important (Arnold et al., 2004; Britter \& Hanna, 2003). A number of relevant wind tunnel and field experiments have been reported in the literature, e.g. Arnold et al. (2004); Cheng \& Robins

$\overline{1}$ Corresponding author. Email: z.xie@soton.ac.uk; tel: +44(0)23 80594493 
(2004); Rotach et al. (2004); Yee \& Biltoft (2004); Carpentieri et al. (2008). Despite their disadvantages compared with experiments, numerical approaches have numerous advantages, e.g. high controllability, lower cost (sometimes!), three-dimensional/four-dimensional data output. However, Britter \& Hanna (2003) point out that computational fluid mechanics studies (i.e. Reynolds Averaged Navier-Stokes methods-RANS) can sometimes produce reasonable qualitative results for mean flows but the performance, when compared with laboratory or field experiments, is little better than that of simple operational models. Quite a few researchers in this area have turned to large-eddy simulation (LES).

Indeed, LES has demonstrated its capability and potential for such applications, e.g. Baik et al. (2007); Baker et al. (2004); Coceal et al. (2006); Hanna et al. (2002); Kanda et al. (2004); Meinders \& Hanjalić (1999); Stoesser et al. (2003); Xie \& Castro (2006); Xie et al. (2008c). All of these LES (and direct numerical simulation - DNS) studies focused on flow over simple geometries, e.g. a group of cubes or different height cuboids with the same square base, or a two-dimensional street canyon. These works use simple periodic in-outlet boundary conditions for which it is assumed that the computational domain is one repeated unit of the whole urban geometry, or a very simple inflow boundary condition without proper correlation in time and in space. Periodic boundary conditions may generate severely biased results for a genuine urban domain with a limited size, e.g. for DAPPLE site (not reported in this paper). Inflow specified at the inlet without proper correlations in time or in space have been shown in the literature to be inadequate, as we confirmed in Xie \& Castro (2008a).

As a reasonable start to gain a deeper insight to the dispersion mechanisms in urban environments, it is useful to investigate the flow over typical patterns of urban geometry, e.g. street canyons (Baik et al., 2007; Baker et al., 2004), staggered, aligned or square arrays of blocks with uniform height (Coceal et al., 2006; Hanna et al., 2002; Kanda et al., 2004; Meinders \& Hanjalić, 1999; Stoesser et al., 2003) or non-uniform height (Xie et al., 2008c). However, there is a scarcity of reliable numerical models for flow and dispersion over a genuine urban geometry for understanding the integrative effect of combinations of these morphological patterns or for practical purposes. There are some reports available in the literature on building-resolving LES for genuine urban geometry. Tseng et al. (2006) used LES to predict flow and dispersion in downtown Baltimore, MD with a domain containing about 30 buildings. The inflow velocity field on the inlet plane was obtained from a separate flow simulation without buildings that uses periodic boundary conditions in the streamwise direction. The buildings were resolved using 6-8 grid points. Such a low resolution may not predict the second-order moment statistics accurately. And there were no experimental data reported for the validation of LES in their paper. More recently, Smolarkiewicz et al. (2007) used LES to simulate dispersion over a 1:200 scale model of the Pentagon building, and compared LES data with their wind tunnel data. A laminar flow was used for 
the inflow boundary condition of the LES computations. It is not clear how serious are the errors such a simple inflow boundary condition generates when the LES data is used for modelling the full scale case. As far as we are aware, no comprehensive LES work for flow and dispersion over a genuine urban site has been performed and validated quantitatively against experiments.

In order to establish the credibility of LES as a tool for operational/practical forecast applications, there are many issues which must be addressed, such as: 1. Is there a general minimum resolution to produce reasonable turbulence statistics? If the answer is 'YES' for the flow, is it also applicable for scalar dispersion? 2. Is LES reliable for high Reynolds number? 3. How much does LES accuracy depend on the mesh resolution and quality? 4. What is the most appropriate inflow boundary condition? 5. Should coupling between the weather scale flow and the urban boundary layer be achieved?

Recently an LES model implemented in major commercial codes was used to calculate the turbulent flow over staggered wall-mounted cubes and a staggered array of random height obstacles with an area density $25 \%$ (Xie \& Castro (2006), hereafter denoted XC). It was found that a significantly coarser mesh (e.g. approximately $20 \times 20 \times 20$ grid points per building block) than required for a full DNS produced sufficiently accurate results. Turbulence generated by urban-like obstacles, e.g. cuboid-shape bodies with sharp edges, is building-block-scale dominated and viscous effects (on the building surfaces, for example) are insignificant, so that for this type of flow the precise wall condition/subgrid-scale model is unimportant for capturing the buildingblock-scale flows and surface drag. The results also confirm that Reynolds number dependency of such flows (if it does exist) is very weak, as suggested also by wind tunnel experiments. Such complex wall flows are thus, paradoxically, significantly easier to compute with LES than are smooth-wall flows, where viscous effects dominate near the wall. These issues are fully discussed in $\mathrm{XC}$.

In the present paper, flow and point source dispersion over a genuine urban geometry - the DAPPLE site (http://www.DAPPLE.org.uk) - were simulated numerically using LES. There were more than fifty building obstacles (including a dome and a tower) in the DAPPLE site. The first issue was how to fulfil the resolution requirement without exceeding the available computer's capability. The mean building height of DAPPLE was 22 meter. As we had previously concluded that roughly $20 \times 20 \times 20$ grids points per building block produces sufficiently accurate results for such flows (see XC), a resolution down to one meter was chosen for the LES. Our objective is to simulate flow and pollutant dispersion within genuine urban canopy regions. Such a localised computational domain may in fact be smaller than the typical city size. This exacerbates the difficulties posed by having to supply appropriate boundary conditions. One requires an efficient method to insert appropriate small-scale turbulence at the upstream boundary at each time step, recognising that the flow is almost never close to being spatially periodic. Our final objective is 
coupling weather-scale computations (for example from the UK Met Office's weather code, the Unified Model - UM) to smaller-scale computations of flow and dispersion within urban environments. This also requires a particularly efficient means of providing dynamically changing turbulence data at the inlet of the computational domain. A new inflow technique (Xie \& Castro, 2008a) has been used for all the LES computations reported here.

In the following sections, firstly a very brief description of the governing equations is given in $§ 2$; secondly, numerical settings including geometry and mesh are given in $\S 3$; thirdly, inflow conditions are given in $\S 4$; fourthly, LES for DAPPLE is validated in $\S 5$; fifthly, $\S 6$ describes numerical experiments performed to investigate the sensitivity to wind direction, inflow condition, source location, etc; finally in $\S 7$ concluding remarks are given.

\section{Governing equations}

To ensure a largely self-contained paper, a brief description of the governing equations of LES is given here. More details can be found in XC. The filtered continuity and Navier-Stokes equations are written as follows,

$$
\begin{aligned}
& \frac{\partial \bar{u}_{i}}{\partial x_{i}}=0 \\
& \frac{\partial \bar{u}_{i}}{\partial t}+\frac{\partial \bar{u}_{i} \bar{u}_{j}}{\partial x_{j}}=-\frac{1}{\rho}\left(\frac{\partial \bar{p}}{\partial x_{i}}\right)+\frac{\partial}{\partial x_{j}}\left(\tau_{i j}+\nu \frac{\partial \bar{u}_{i}}{\partial x_{j}}\right) .
\end{aligned}
$$

The dynamical quantities, $\bar{u}_{i}, \bar{p}$ are resolved-scale (filtered) velocity and pressure respectively. $\nu$ is the kinematic viscosity. In the near-wall region, the Lilly damping function was also applied. $\tau_{i j}$ is the subgrid-scale (SGS) Reynolds stress. The Smagorinsky SGS model was used, $\tau_{i j}-\delta_{i j} \tau_{k k} / 3=2 \rho\left(C_{s} \Delta\right)^{2}$ $\left(2 \bar{s}_{m n} \bar{s}_{m n}\right)^{1 / 2} \bar{s}_{i j}$, where $\bar{s}_{i j}=\frac{1}{2}\left(\frac{\partial \bar{u}_{i}}{\partial x_{j}}+\frac{\partial \bar{u}_{j}}{\partial x_{i}}\right) ; \Delta$ is taken as the cubic root of the cell volume; $C_{s}=0.1$, which is recommended by Shah (1998) for flow past a blunt obstacle. $\tau_{k k}$ is modelled according to a closure similar to the one devised by Yoshizawa (1986). $\delta_{i j}$ is the Kronecker-delta. In our previous paper - XC, we argue because such flows are block-scale dominant so that, provided the grid is sufficiently resolved to capture much of the inertial range in the spectrum, the LES results are insensitive to the precise form of the SGS model.

For cases where the fine eddies in the vicinity of the wall are important, it is recommended that $\mathscr{N}_{1}^{+}$is of order of unity $\left(\mathscr{N}_{1}^{+}\right.$is the distance in wall units between the centroid of the first cell and the wall assuming the $\mathscr{N}$ coordinate is normal to the wall). Note, however, that for a complex geometry, where separation and attachment processes occur, it is impossible to satisfy this 
criteria everywhere. We argue that, unlike the situation for smooth-wall flows, it is in fact not necessary, at least for obtaining overall surface drag and the turbulent motions at the scale of the roughness elements (buildings), which turn out to be dominant (see XC).

The filtered governing equation for the scalar is written as follows,

$$
\frac{\partial \bar{c}}{\partial t}+\frac{\partial \bar{u}_{j} \bar{c}}{\partial x_{j}}=\frac{\partial}{\partial x_{j}}\left[\left(K_{s}+K_{m}\right) \frac{\partial \bar{c}}{\partial x_{j}}\right]+S
$$

where $K_{s}$ and $K_{m}$ are the subgrid turbulent diffusivity and molecular diffusivity respectively and $S$ is the source term. Up to now most studies for concentration dispersion problems have applied a subgrid eddy viscosity combined with a subgrid scale Schmidt number, which are set as constant or calculated dynamically. In the present study, we adopt this approach using a constant subgrid scale Schmidt number of unity. $K_{m}$ is small in our simulation, since the Reynolds number is large, but that term is nevertheless included.

\section{$3 \quad$ Numerical settings}

The entire LES model was implemented in the code described in XC. Crucially, the discretisation for all terms in Eq. $(1,2)$ was second order accurate in both space and time. The convective terms in Eq. $(1,2)$ were discretized in space using the 2nd order monotone advection and reconstruction scheme (MARS). An efficient inlet turbulence generation scheme (Xie \& Castro, 2008a) was used, with symmetric conditions at the lateral boundaries and a stress-free boundary at the top $\left(z=10 h_{m}\right.$, where $h_{m}$ is the mean height of the buildings in the domain - $110 \mathrm{~mm}$ in the 1:200 scale model used, see below). The top boundary condition here is simple but widely used in literature, e.g. Moeng (1984). The artificial boundary condition may be interpreted as a strong inversion. A detailed setting of the inflow approach can be found in $\S 4$. The Reynolds number based on $h_{m}$ and the free stream velocity $U_{r e f}$ was approximately 18,000 . The time step was $0.003125 \mathrm{~s}$, giving an averaged CFL number of 0.48 , and the initial duration of most of the runs was $62.5 \mathrm{~s}$ with subsequent averaging over at least $125 \mathrm{~s}$ for all the statistics.

\subsection{Geometry, mesh and resolution.}

A description of the DAPPLE field experiment can be found in Arnold et al. (2004). Fig. 1 shows the schematic plan view of the 1:200 scale wind tunnel model of the site. Block heights are shown on each. Note the two coordinate systems used $-x_{t}, y_{t}, z$ is the model system with the $x_{t}$ axis from west to 


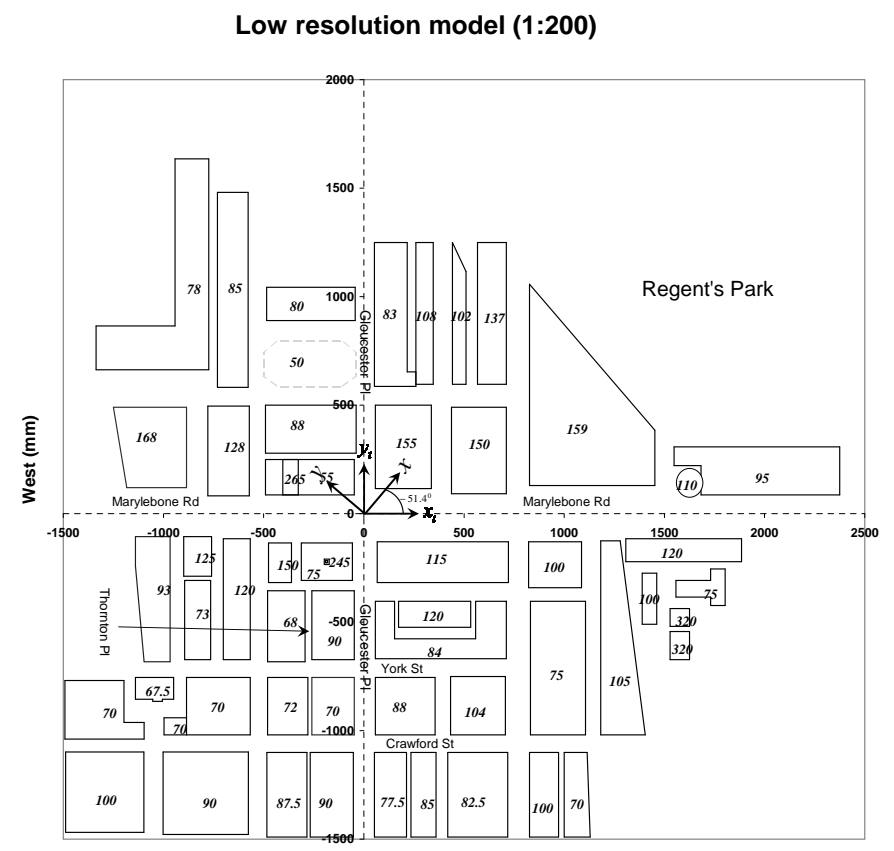

Fig. 1. Plan view of the wind tunnel model. Numbers in italics on each building block indicate its height in mm. The model coordinates are marked in mm, with $x_{t}$ from west to east, $y_{t}$ from south to north and $z$ from ground to top respectively. $x, y, z$ are the computational coordinates (Fig.2).

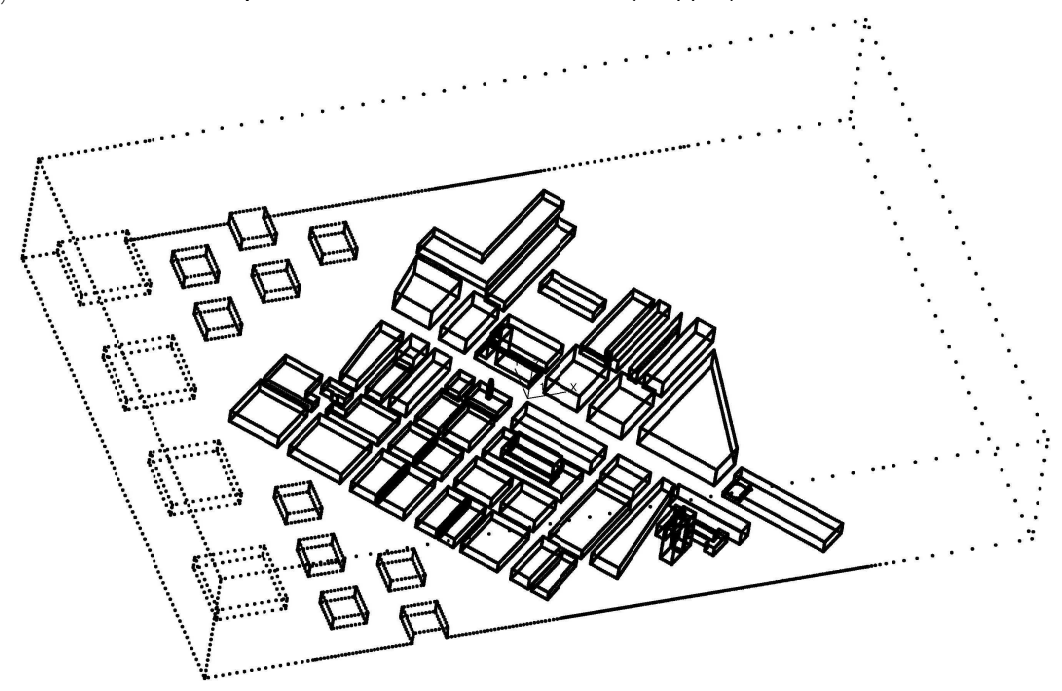

Fig. 2. Computational domain, with the coordinate origin at the ground at the major intersection; its size is $L_{x}=6000 \mathrm{~mm}, L_{y}=4000 \mathrm{~mm}, L_{z}=1000 \mathrm{~mm}$.

east, $y_{t}$ axis from south to north and $z$ axis from ground to top respectively, whereas $x, y, z$ is the computational one, with the $x$ axis from the inlet to the outlet corresponding to the wind tunnel axis(the wind direction), and the $y$ axis following the right-hand rule respectively (with $z$ vertical).

Fig. 2 shows the computational domain, which is equivalent to $1200 \mathrm{~m}$ (streamwise) $\times 800 \mathrm{~m}$ (lateral) $\times 200 \mathrm{~m}$ in full scale. Note the computations were conducted at wind tunnel size and all the sizes are given in $\mathrm{mm}$ if not explicitly denoted. The mean height of the model buildings $\left(h_{m}\right)$ is $110 \mathrm{~mm}$ and their 


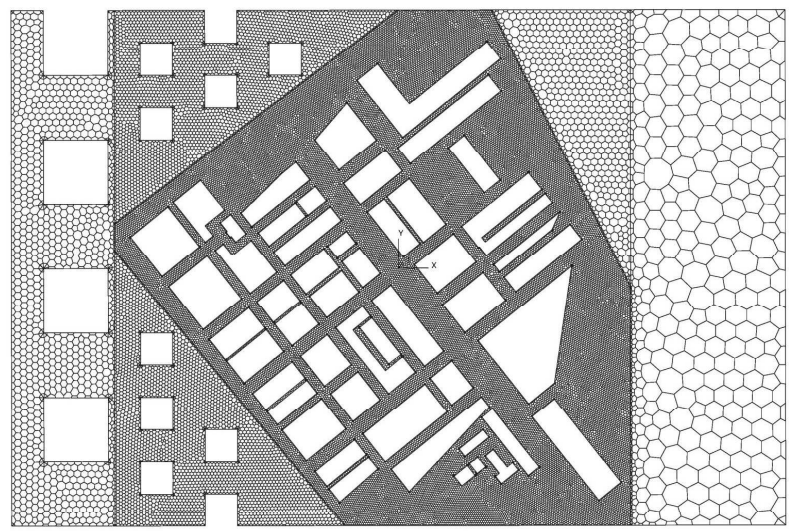

(a)

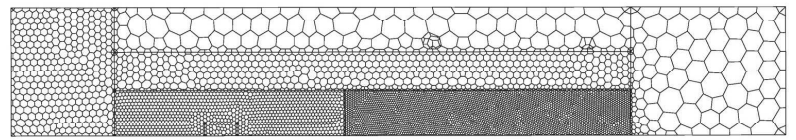

(b)

Fig. 3. Polyhedral mesh. (a) a horizontal plane at $\mathrm{z}=0$; (b) a vertical plane at $\mathrm{y}=-2 \mathrm{~m}$.

packing density is $0.5 \pm 0.001$ (Padhra et al., 2008). Because of the lack of geometrical information upstream of the DAPPLE site, three rows of staggered 'artificial' blocks with height $110 \mathrm{~mm}$ were installed in the near inlet region. Correspondingly, in the EnFlo wind tunnel a large number of two-dimensional roughness elements were installed upstream of the DAPPLE site. The artificial blocks were useful to retain a laterally averaged mean velocity profile not too dissimilar in shape to the one imposed at the inlet, which was obtained over the two-dimensional roughness elements in the wind tunnel. Numerical experiments on the sensitivity of the arrangement of the artificial blocks to flows and scalar dispersion at the intersection of Marylebone Rd and Gloucester Pl, hereafter denoted the major intersection, were performed. The gaps between blocks one and two, two and three, and four and five (counting from right to left looking downstream, see Fig.2) in the second row were entirely blocked by inserting thin solid walls with height $h_{m}$ in the gaps. The other settings were kept unchanged. The turbulence data and the scalar data were compared with those in $\S 5$ and it was found that the differences were negligible. This suggests that the flow and the scalar dispersion within the DAPPLE site were not too sensitive to the arrangement of the appropriate artificial blocks when a proper inflow condition with correlations in space and in time is imposed. No artificial building blocks were installed in the near outlet region where, downstream of the DAPPLE site, London's Regent's Park was situated.

In order to simulate the flows over a genuine urban canopy with a very complex geometry, unstructured non-hexahedral meshes inevitably have to be used. Tetrahedral meshes are widely used in CFD because methods which do this are mature, efficient and highly automated (Peric, 2004). Perhaps not surprisingly, it was found that the accuracy of tetrahedral meshes for LES of these urban-type flows, even at a higher resolution, is not so high as that of uniform 


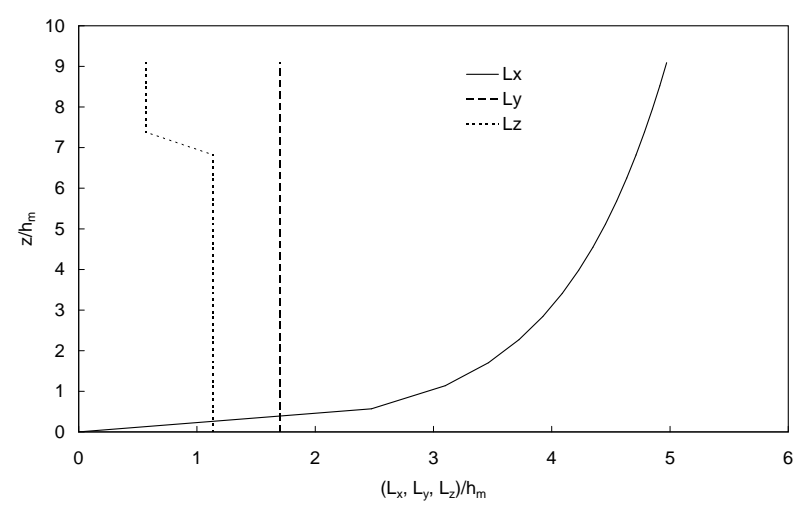

Fig. 4. Vertical profiles of prescribed integral length scales for LES at inlet.

hexahedra meshes (for equal computational effort) (Xie \& Castro, 2008b). Furthermore, unstructured polyhedral meshes offer substantially better properties than tetrahedral meshes (Peric, 2004). A major feature of polyhedral meshes is that the simplest (and most efficient) approximations that are used on Cartesian meshes (e.g. linear interpolation, midpoint rule) are applicable and provide nearly the same accuracy as on Cartesian meshes. The flexibility of polyhedral approach is that the mesh can be optimized in many ways to satisfy the critical requirements of achieving second order accuracy (e.g. the line connecting two neighbour cell centroids should pass through the centroid of the joint face). Polyhedral meshes in the present case were validated for flow over uniform cubes and then a more random geometry (Xie et al., 2008c).

Fig.3 illustrates how the DAPPLE domain (as shown in Fig.2) was discretized into polyhedral cells with three level resolution in the vertical direction and four level resolution in the streamwise direction. In Fig.3b, the ratios of the grid size at the first, second and third vertical interfaces (from the inlet to the outlet) for $z<3 h_{m}$ were approximately $2,1.4$ and 0.5 respectively. The first and the second interfaces were both upstream of the DAPPLE site, whereas the third one was downstream. The ratios at the first (i.e. at $z=3 h_{m}$ ) and the second (i.e. at $z=6 h_{m}$ ) horizontal interfaces, which are all above the DAPPLE site, were approximately 2.5 and 2 respectively. The size of the smallest cells, which were those cells nearest the walls, was approximately $h_{m} / 15$. The 2nd order monotone advection and reconstruction scheme (MARS) was used to eliminate any possible numerical instabilities. We emphasize that the DAPPLE region itself was meshed essentially uniformly up to a height of $3 h_{m}$ and the modest discontinuity in mesh sizes elsewhere do not have a significant effect on the solution accuracy.

\section{Inflow conditions}

Simulating the flows over a genuine urban region, in particular using a numerical weather forecasting code (e.g. the UK Met Office's Unified Model) to 

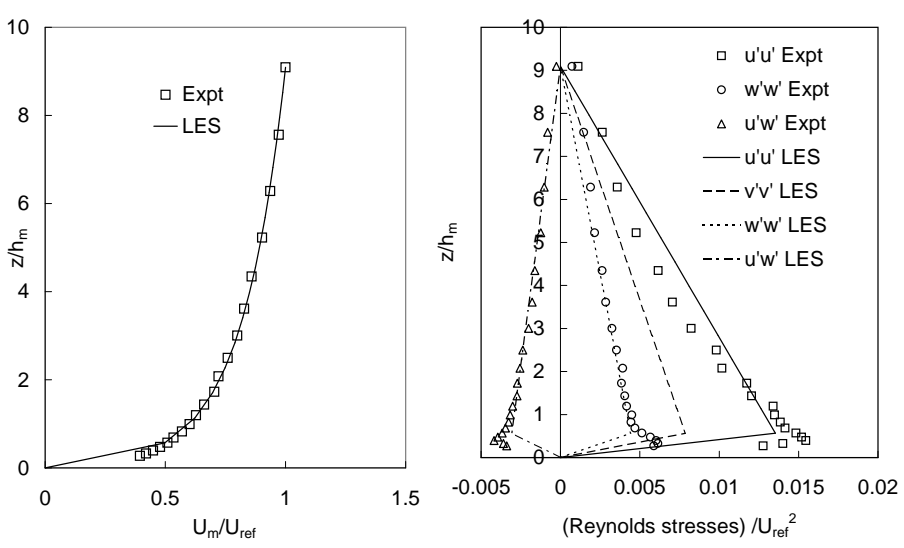

Fig. 5. Vertical profiles of measured (symbols) and prescribed (lines, for LES at inlet) mean velocity and Reynolds stresses.

provide the dynamic large scale inlet boundary conditions, requires a continuous specification of appropriate inlet turbulence. For such computations to be practical, a very efficient method of generating such turbulence is needed. A digital-filter-based generation of turbulent inflow conditions has been developed and is reported in Xie \& Castro (2008a). The artificially generated turbulent inflows satisfied prescribed integral length scales and a prescribed Reynolds stress-tensor.

The inlet integral length scales, which were estimated from Cheng \& Castro's cube cases (Castro et al., 2006), are shown in Fig.4. $L_{x}, L_{y}$ and $L_{z}$ are the integral length scales of the axial velocity component in the streamwise, lateral and vertical directions respectively. The mean velocity and the Reynolds stresses at the inlet were obtained by fitting the measured data from the DAPPLE wind tunnel experiments (Cheng \& Robins, 2004) and are shown in Fig. 5, where $U_{r e f}$ is the free stream velocity. Note that Xie \& Castro (2008a) showed that the computational results are not too dependent on the precise nature of the prescribed length scale profiles, although it is very important that they be non-zero so that the inlet turbulence has genuine spatial structure.

\section{Numerical results and data analysis}

\subsection{Turbulent flow over the DAPPLE site}

From henceforth, all numerical values for specific locations are given in mm, unless otherwise stated. Attention is focused on one wind direction (i.e. $-51.4^{\circ}$ bearing clockwise to the Marylebone Rd direction - approximately southwest wind). The numerically simulated mean velocity and Reynolds stress profiles at fourteen typical stations in the near region of the major intersection (i.e. within $\leq 40 \mathrm{~m}$ in full scale) were found to be in good agreement with those from the wind tunnel experiments. Fig. 6 shows typical examples of vertical 

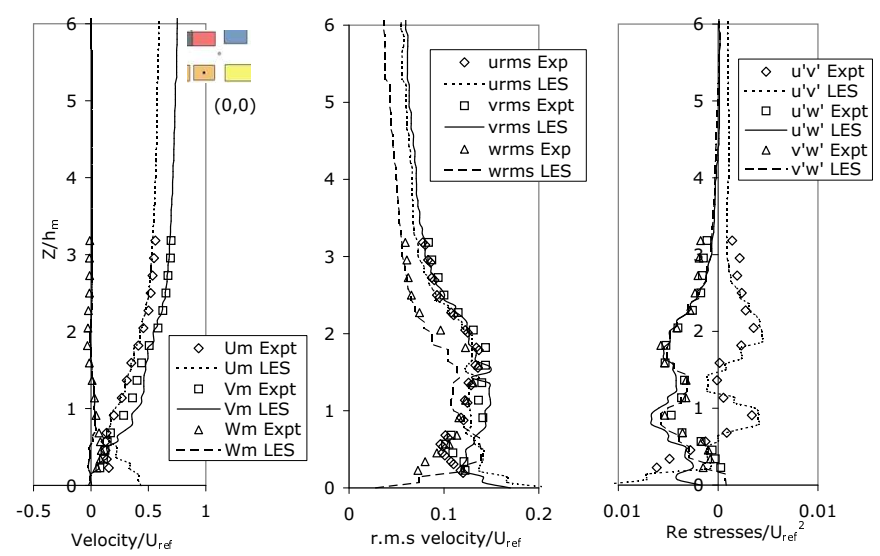

Fig. 6. Vertical profiles of time-mean velocities, velocity fluctuation r.m.s, Reynolds shear stresses in the model coordinate $\left(x_{t}, y_{t}, z\right)$ at the major intersection.
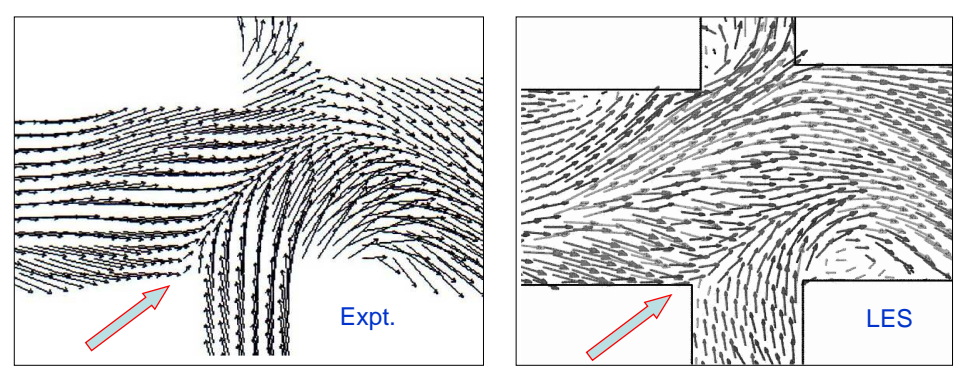

Fig. 7. Time-mean velocity vectors at $\mathrm{z}=0.23 h_{m}$ on the major intersection.

profiles of time-mean velocities, velocity fluctuation r.m.s, and Reynolds shear stresses in the model coordinates $\left(x_{t}, y_{t}, z\right)$ at the major intersection.

Figure 7 shows a comparison between the LES and the wind tunnel experiment of the time-mean velocity vectors $\left(U_{m}, V_{m}\right)$ on a horizontal plane $z=0.235 h_{m}$ at the major intersection. The separation bubble on the corner of the southeast building was successfully predicted using LES. However, it was found that steady Reynolds Averaged Navier-Stokes equations (RANS) models using the same mesh as that for LES failed to predict this separation bubble, which confirms previous remarks on steady RANS models for such flows, e.g. in XC.

Six 3-D mean streamlines starting at $x_{t}^{s}=-356, y_{t}^{s}=-702$ and $z^{s}=0,10,20$, 30, 40 and 50 are shown in Fig.8. Here, a streamline is the path that a particle of zero mass would take through the fluid domain driven by the mean velocity vector field. The path was calculated using a Runge-Kutta method. Helicaltype streamlines are quite evident. The six pathlines separate significantly after a short distance, e.g. $100 \mathrm{~mm}$, which might suggest that the transport of the scalar is sensitive to the source height. However, glancing ahead to Fig.14 shows that a large portion of the mean concentration cloud released from the lowest point source $\left(z^{s}=10\right)$ turns left (looking downstream) at the first downstream intersection (i.e. York St-Thornton Pl) into the narrow street - Thornton Pl. This suggests that we cannot expect to interpret too 


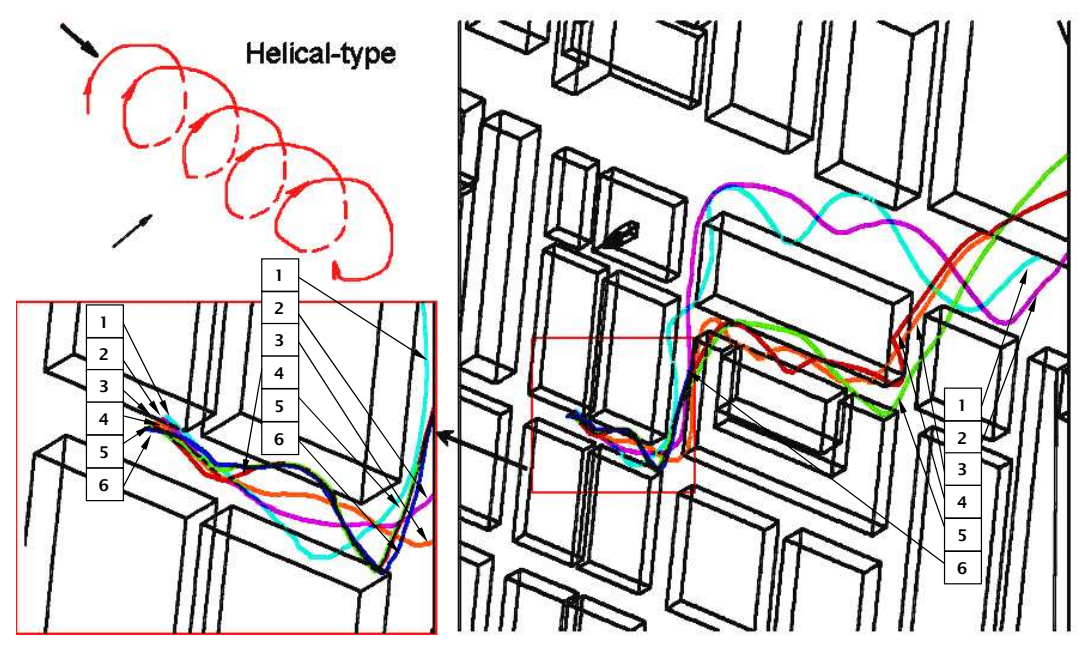

Fig. 8. Time averaged pathlines released at $x_{t}^{s}=-356, y_{t}^{s}=701.9, z^{s}=0,10,20$, 30,40 and 50 .

much from the 'mean' pathlines, which don't of course include any influence of the turbulent diffusion processes.

Spatially averaged mean velocity and Reynolds stress profiles were obtained over the region $-1000<x_{t} \leq 1000,-1000<y_{t} \leq 1000$ (in which the packing density is about 0.53 - slightly greater than that of the whole DAPPLE site) and are shown in Fig. 9. The peaks of $u_{r m s}, v_{r m s}, w_{r m s}$ and $\left\langle u^{\prime} w^{\prime}\right\rangle$ occured at the height $1.5 h_{m}$, not at the mean building height $h_{m}$. Note that in this region (see Fig.1), there were five large and tall buildings with heights around $1.5 h_{m}$. This confirms the previous finding, e.g. in Xie et al. (2008c), that peaks occur at the height of the tallest building. Note that it has also been found that the tallest buildings provide the dominant contributions to the total drag force. In the canopy region the temporal r.m.s. velocities are of the same order as the mean velocity, which is again consistent with the results in Xie et al. (2008c), where flows over a group of staggered cubes or random height blocks were investigated. The spatially averaged cross-wind velocity within and immediately above the canopy is small here, which might be because of the random nature of the arrangement of the building blocks.

\subsection{Point source dispersion}

Five different non-reactive tracers were released at five typical stations - S1 $(-391,-725,10), \mathrm{S} 2(-356,-702,10), \mathrm{S} 3(0,-600,10), \mathrm{S} 4(0,-500,10)$ and S5 $(-356,-1060,10)$, which were all upstream of the major intersection shown in Fig.10; numbers in brackets $\left(x_{t}, y_{t}, z\right)$ refer to the model coordinates of each source. Fig.10 shows a comparison between LES and wind tunnel data for the mean, normalised concentration at the ten R-locations indicated, for the source at S2. The coordinates of the R-locations are shown in Table 1. Here the medium mesh is the mesh shown in $§ 3.1$. For the fine mesh, in a rectangular 

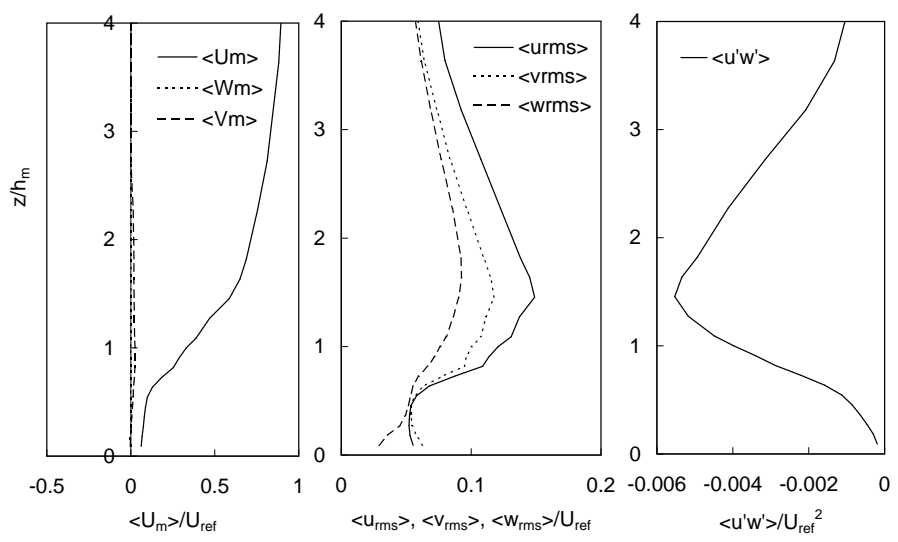

Fig. 9. Vertical profiles of spatially averaged mean velocity, velocity r.m.s. and Reynolds shear stress profiles in the computational coordinates $x, y, z$.

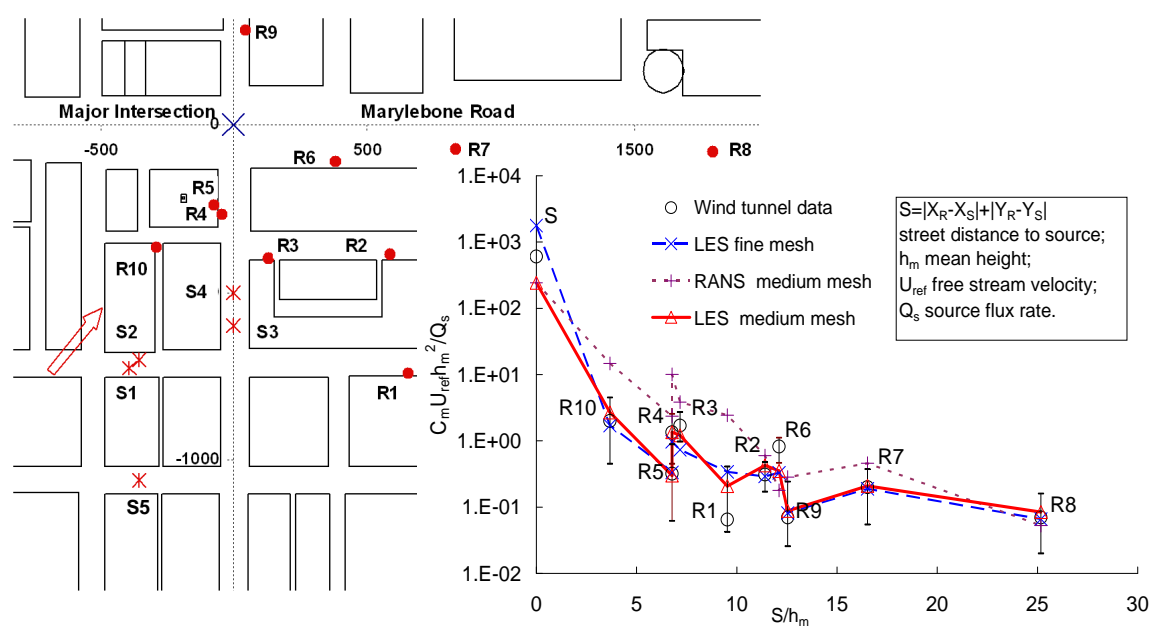

Fig. 10. Mean concentration for LES and concentration for RANS at ten DAPPLE stations R1-R10, for which the coordinates are given in Table 1, from source at S2.

Table 1

Coordinates in mm of the ten DAPPLE stations R1-R10.

\begin{tabular}{c|cccccccccc}
\hline station & $\mathrm{R} 1$ & $\mathrm{R} 2$ & $\mathrm{R} 3$ & $\mathrm{R} 4$ & $\mathrm{R} 5$ & $\mathrm{R} 6$ & $\mathrm{R} 7$ & $\mathrm{R} 8$ & $\mathrm{R} 9$ & $\mathrm{R} 10$ \\
\hline$x_{t}$ & 654 & 587 & 130 & -43 & -72 & 385 & 832 & 1793 & 43 & -288 \\
$y_{t}$ & -740 & -389 & -399 & -269 & -240 & -111 & -72 & -82 & 279 & -365 \\
$z_{t}$ & 7.5 & 7.5 & 7.5 & 7.5 & 82.5 & 7.5 & 7.5 & 7.5 & 7.5 & 7.5
\end{tabular}

region $\left(-700<x_{t} \leq 500\right.$ and $\left.-800<x_{t} \leq 200\right)$ the cell size was reduced to two thirds of that of the medium mesh and in the very-near region of the point source the cell size was further reduced to approximately one third of that of the medium mesh.

Except in the near source region, the results from the medium mesh computation were in good agreement with those from the fine mesh, which suggests a good degree of mesh independence. The discrepancy in the near source region is almost certainly due to the effect of the source size, which was effectively 

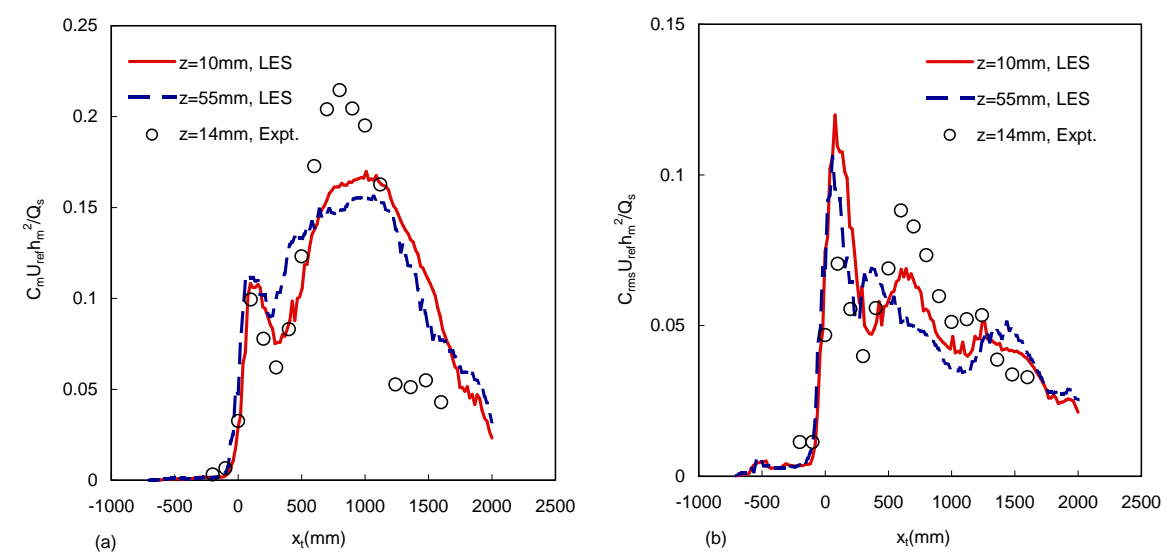

Fig. 11. Dimensionless mean and r.m.s. concentration along Marylebone Rd (i.e. along $\left.y_{t}=0\right)$. The source location is $\mathrm{S} 5$ and $Q_{s}$ is the source flux.

one grid size. Both sets of data are in reasonable agreement with the measurements, with values of concentration falling four decades from the source to station R8. However, at station R1 LES over-predicts the mean concentration. Note that in the absence of the buildings, R1 was the most remote station from the plume core in terms of the ratio of the distance and the local plume width. At the remote edge of the plume, very high intermittency of the concentration signals occurs; this requires time-averaging durations longer than those used if fully converged values are required.

A standard $k-\epsilon$ model was also used to predict point source dispersion over the site. The same domain shown in Fig. 2 and the medium mesh were used. The inlet velocity, Reynolds stresses and length scales shown in Fig. 5 and 4 were used to generate appropriate input profiles for the $k-\epsilon$ model. Fig. 10 includes a comparison between LES and RANS of the predicted concentration, in which the $k-\epsilon$ model generally over-predicts the concentration, sometimes by an order of magnitude, at the ten R-locations. It was found that, (1) on the vertical cross section through the source and along the wind direction, RANS underestimated the concentration above the urban canopy but overestimated it within the canopy; (2) on a ground level horizontal cross section, RANS predicted null concentration over a narrow street downstream of the source, whereas LES did not. This is probably partly due to the RANS' weakness in prediction of a genuinely unsteady separation bubble at the corner of buildings.

Mean and fluctuating concentration along the main streets were also compared with measurements. Fig.11 is a typical example. Note that the tracer was released at S5 (see Fig.10). In the absence of the buildings, the plume core crossed the Marylebone Rd approximately at $x_{t}=500$. The locations of the peak mean concentration at heights $z_{t}=110$ (not shown here), 55 and 10 are approximately $x_{t}=600,800$ and 1000 respectively. This suggests that within the canopy the buildings affect the location of the peak mean concentration more than above the canopy, which is perhaps not surprising. The eastward shift of the location of the peak mean concentration could well be due to a channelling effect (i.e. along Marylebone $\mathrm{Rd}$ ). At the major inter- 
section a secondary peak of mean concentration occured, which is due to the high concentration clouds coming from upstream along Gloucester Pl. Fig.11b shows a different pattern of the peaks - the strength of the peak of $C_{r m s}$ at the intersection was at least comparable with that at the core of the plume (i.e. approximately at $x_{t}=600$ ). This could be due to the high intermittency of the concentration signal at the intersection.

\section{$6 \quad$ Numerical experiments}

\subsection{Sensitivity to source location and inlet conditions}

Sensitivity of the local and far field mean concentration to the source location was investigated extensively. Concentrations arising from a source at S1 were compared with those from a source at S2. The former is in the middle of York $\mathrm{Rd}$, whereas the latter is very close to the building on the north side, as shown in the inset in Fig.12. The mean concentration profiles over Marylebone Rd are essentially the same for the two sources. Note that in the absence of the buildings the plume centre crossed the Marylebone $\mathrm{Rd}$ at $x_{t}=200$. It is hard to discern any difference between the two peaks shown in Fig.12a. The $c_{r m s}$ profiles shown in Fig.12b have the same pattern for the two sources, but some quantitative discrepancy.

Despite the similarities along the Marylebone Rd, significant differences were noted in the near source regions. Fig.13 shows the local behavior of the concentration dispersion for source S1. The high concentration clouds from S1, which was located in the middle of the road, were driven to the leeward wall due to the helical-type circulation flow in the street canyon shown in Fig.8. The scalar is thus more likely to be convected out of the canyon. Subsequently, the concentration is low on the intersection immediately downstream. Fig.14 shows, on the other hand, the local behaviour of the concentration dispersion for source S2, which was located close to the windward wall on the north side. Note that the convection effect of the helical-type circulation flow on the concentration transport was less strong than that for the S1 source location. Less plume was ventilated out of the street canyon and consequently a high concentration occurred at the intersection (York St - Thornton Pl) immediately downstream. Very high concentration was found in the narrow street -Thornton Pl - toward the north.

Fig.15 shows a comparison of mean and r.m.s. concentration along Marylebone $\mathrm{Rd}$ for sources S3 and S4. The mean concentration profiles were essentially the same for the two source locations, except a quantitative discrepancy, e.g. at the major intersection. The two $c_{r m s}$ profiles were again essentially the same. Fig.16 and Fig. 17 show the local dispersion behaviour for source S3 and S4 source locations, respectively. Although both S3 and S4 are located in 


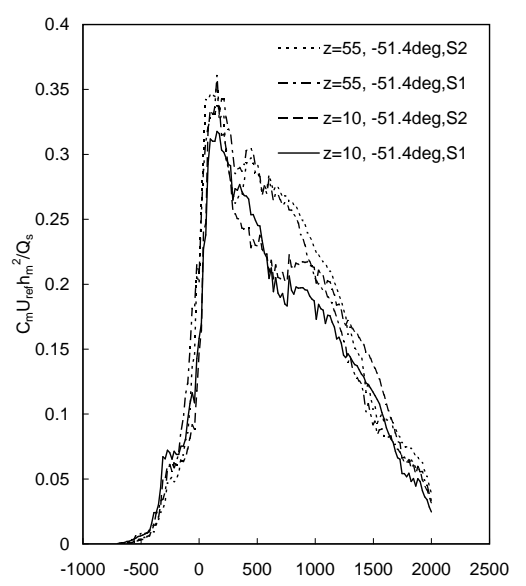

(a)

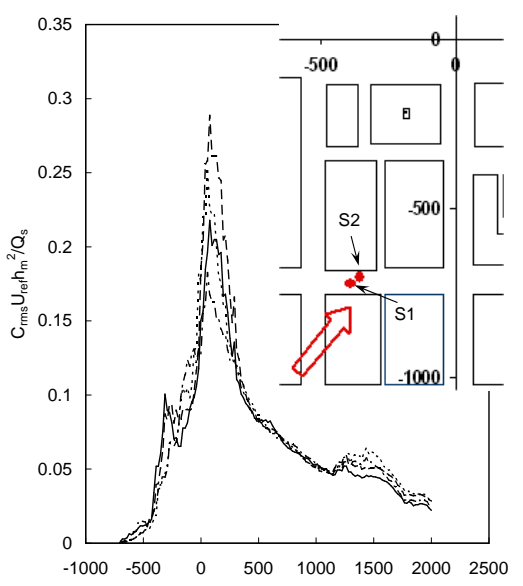

(b)

$\mathrm{x}_{\mathrm{t}}(\mathrm{mm})$

Fig. 12. Sensitivity to the location of the ground level sources at York St on dimensionless mean and r.m.s. concentration along Marylebone Rd.

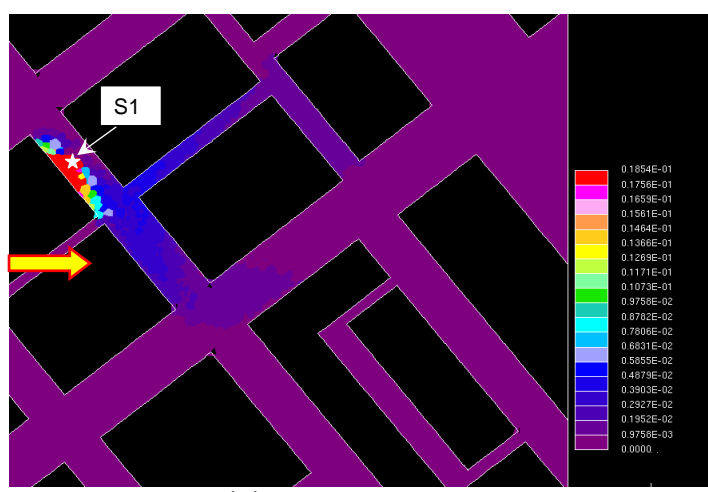

(a)

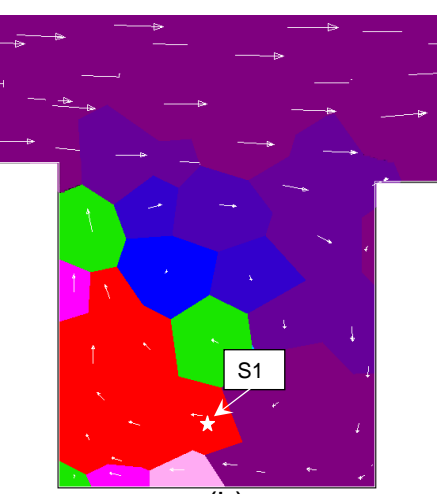

(b)

Fig. 13. Mean concentration and wind vectors in the region near a source at S1. (a) a horizontal plane crossing S1; (b) a vertical plane crossing S1 and perpendicular to the York St.

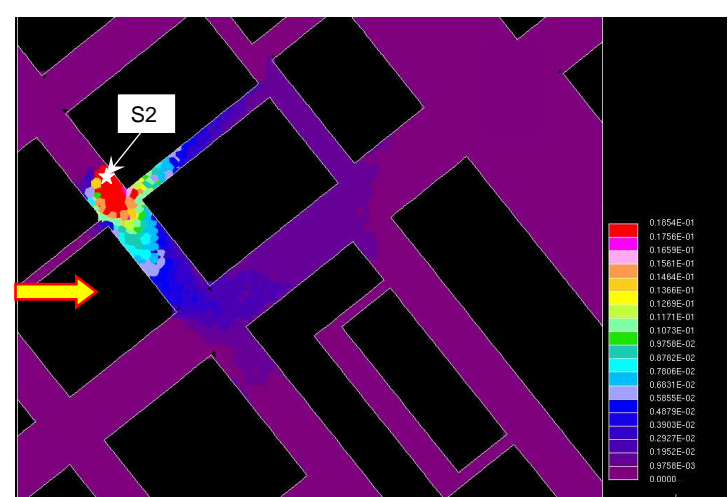

(a)

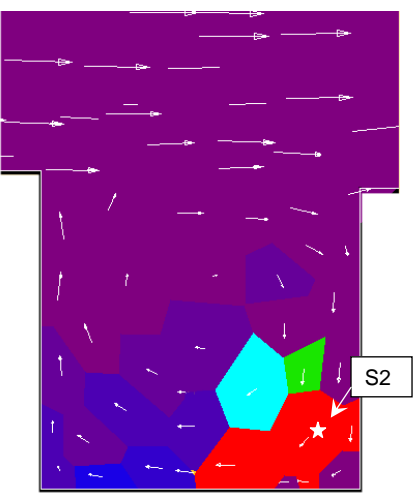

(b)

Fig. 14. As Fig.13, but for S2.

the middle of Gloucester $\mathrm{Pl}$, the patterns of the contours are quite different for the two sources. For S3, the high concentration clouds were evidently transported towards the south (upstream) and also towards the west and then upwards when approaching a building wall. This was partially due to the effect of the immediate upstream intersection (York St - Goucester Pl). For S4, the dispersion process was dominated by the street canyon effect with 

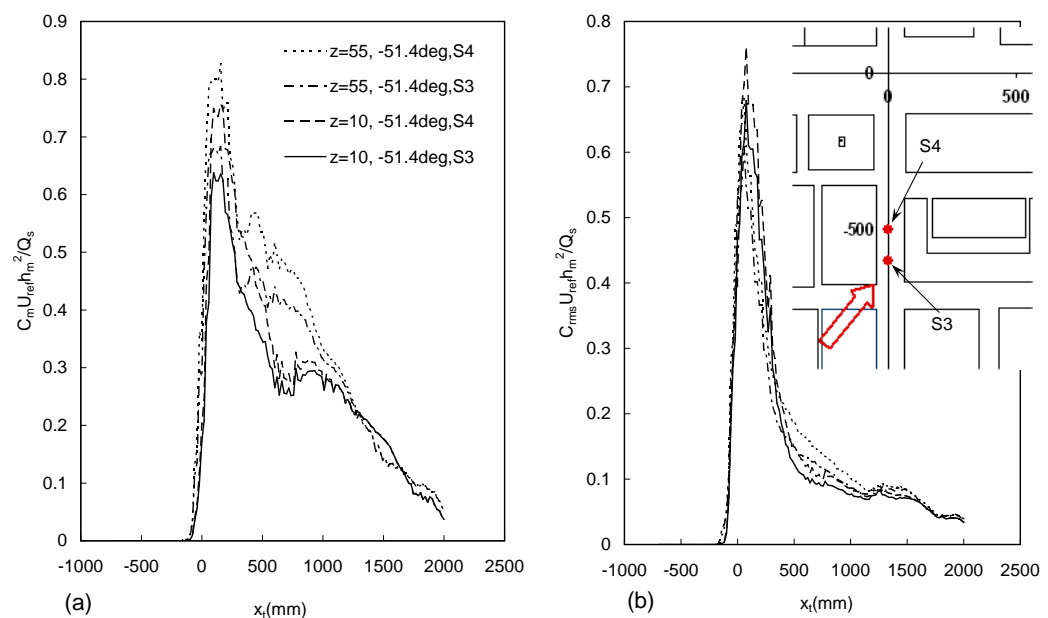

Fig. 15. Sensitivity of the location of the ground level sources at Gloucester $\mathrm{Pl}$ on dimensionless mean concentration and concentration r.m.s. along Marylebone Rd.

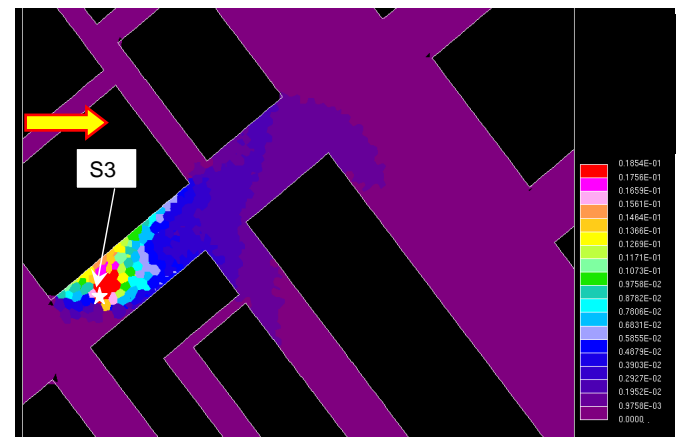

(a)

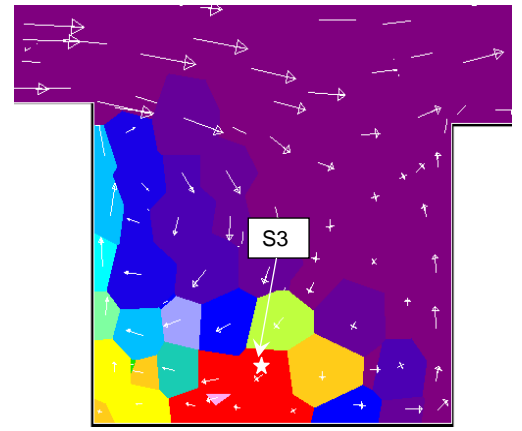

(b)

Fig. 16. Mean concentration and wind vectors in the near region of source S3. (a) a horizontal plane through the source; (b) a vertical plane through the source and perpendicular to Gloucester $\mathrm{Pl}$.

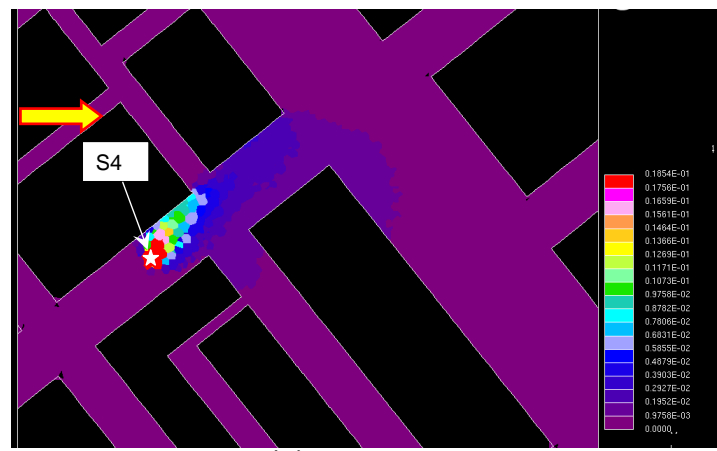

(a)

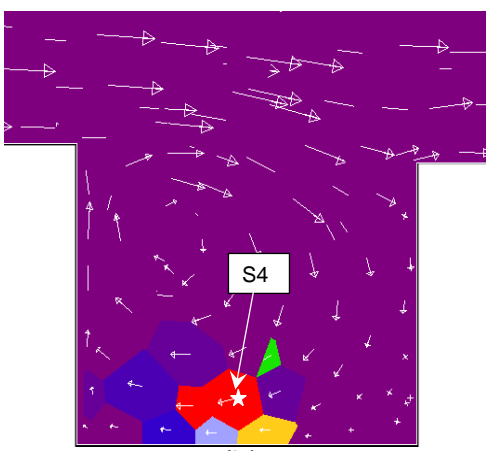

(b)

Fig. 17. As Fig.16, but for S4.

weak convection towards the south. Interestingly, the plume width in the cross street direction for S4 is much less than that for S3 (see Fig.16b and Fig.17b), which might be because the along-street flow was stronger at the lower (S4) elevation than at S3. 


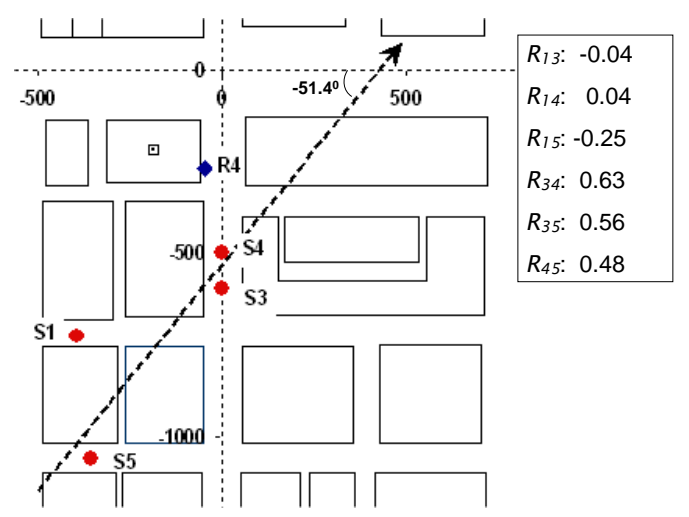

Fig. 18. Sketch of the locations of the sources S1,S3,S4,S5 and the receptor R4, and correlation coefficients of concentration fluctuations at R4.

\subsection{Correlation coefficient of two tracers}

Study of the correlation coefficients arising from two (separately identifiable) tracers released from different continuous sources is very helpful for understanding dispersion from a line source - e.g. from moving vehicles. It is also useful for studying the chemical reaction of two species in the atmosphere. The correlation coefficient can be either positive or negative, enhancing or reducing (respectively) the total concentration fluctuation variance from the combined sources, assuming the sources are of the same species. Four different non-reactive tracers, $c_{1}, c_{3}, c_{4}$ and $c_{5}$, were released simultaneously from S1 (on York St), S3, S4 (on Gloucester Pl) and S5 (on Crawford St), respectively. Time series of the four tracers were recorded at station R4 (on Gloucester Pl - see Table 1 and Fig.18). The correlation coefficient of concentration fluctuations of two tracers (e.g. $m \& n$ ) can be defined as $R_{m n}=\overline{c_{m}^{\prime} c_{n}^{\prime}} / \sqrt{\overline{c_{m}^{\prime 2}}} \overline{c_{n}^{\prime 2}}$, where overbars indicate time averages as usual and $c_{m}^{\prime}, c_{n}^{\prime}$ are the concentration fluctuation of tracers from sources at $S_{m}$ and $S_{n}$, respectively. Fig.18 gives values of the resulting correlation coefficients.

$R_{34}$ is quite high, at 0.63 , which is not surprising as S3 and S4 were only $100 \mathrm{~mm}$ apart. However both $R_{13}$ and $R_{14}$ are very low, probably because of the large angle between the line S1-S3 (or S4) and the wind direction, and neither station $\mathrm{S} 3$ nor S4 is in the core of the plume from source S1. Note that, considering the channelling effect of Gloucester Rd, the local wind direction within the canopy turns anti-clockwise a little for the $-51.4^{\circ}$ direction, looking down in Fig.1, and also there is a tall and large building block located between $\mathrm{S} 1$ and S3. $R_{35}$ and $R_{45}$ are a little lower at 0.56 and 0.48 , respectively. However, note that the street distance between S5 and S3 or S4 is greater than that between $\mathrm{S} 1$ and S3 or S4. It should also be noted that stations S3 and S4 are both in the core of the plume from source S5 (again considering the channelling effect of Gloucester $\mathrm{Pl}$ ), which suggests that concentration signals from sources S5 and S3/S4 are correlated by eddies along their streamwise axes; the turbulence integral length scale in the streamwise direction is a few times greater than 
those in cross wind directions. On the other hand, plumes from S1 and S5 alternatively pass the station $\mathrm{R} 4$ due to the plume meandering driven by large eddies and the channelling effect of the Gloucester $\mathrm{Pl}$, and this generates a negative correlation coefficient $R_{15}=-0.25$.

In their field experiments Hamilton (2007) investigated the correlation coefficients of concentration signals from twin sources on Marylebone Rd, with receptors placed downwind up to three rows of blocks from the sources, and with wind directions $-90^{\circ}$ or $90^{\circ}$ - both perpendicular to Marylebone Rd. In their results, the separation scales of the correlation coefficient profiles were on the order of the mean building height. In the current LES with the sources located on different streets and with wind direction $-51.4^{\circ}$, the behaviour of the correlation coefficients of concentration signals from two sources presents a more complicated picture, emphasising that such results are strongly dependent on local geometry and the specific wind direction.

\section{Final Discussions and Conclusions}

From the above, we draw six major conclusions. (1) LES with a resolution down to one meter in space and one second in time at full scale can give reasonable turbulence and scalar statistics of urban-type environments. (2) It is crucial to have a proper inflow generator for such situations; other computations (not shown here) indicated conclusively that mean \& fluctuating velocities are quite different from those obtained using inadequate temporal and spatial boundary conditions. Indeed, we have also tried using a periodic in-outlet boundary condition for this geometry and we found the LES results were not in reasonable agreement with the wind tunnel data. The problem might be due to the model formulation, e.g. the domain length and domain depth. For example, at the outlet London's Regent's Park (see Fig.1) is situated, where the flow profile must immediately adjust to a profile with smaller roughness length. Furthermore, the inflow conditions adopted in the LES are also more similar to that of the wind tunnel model, where upwind of the DAPPLE site, a large number of two-dimensional roughness elements were installed. (3) However, subject to a proper estimation of mass flow at the inlet within the canopy region, an accurate specification of inlet turbulence fluctuations below the mean building height is not crucial. So, for example, the flow and the scalar dispersion results in the near region of the intersection of Marylebone Rd and Gloucester Pl were not sensitive to the arrangement of the arrays of artificial blocks installed upstream of the DAPPLE site. (4) The flow details within and immediately above the canopy layer are highly dependent on the arrangement and the size of local individual blocks. (5) The scalar dispersion in the near field (i.e. within a distance of $\mathrm{O}(h)$ of the source) is sensitive to the location of the source, whereas in the far field it is not. (6) The correlation coefficient of the concentration signals from two sources at different locations, at least for a wind direction of $-51.4^{\circ}$, presents a com- 
plicated picture whose details depend significantly on the local geometry and the wind direction. Our main conclusion is that LES, with an appropriate inflow technique, can produce satisfactory and affordable simulations of flow and scalar dispersion within and above usefully-sized sub-domains of a city region. There is no reason to suppose that these conclusions would not hold for any other specific urban conurbation.

Acknowledgment: This project was supported by NERC under the Urban Meteorology theme of the Universities Weather Research Network (UWERN, Grant No. DST/26/39 and, more recently, NCAS, Grant No.R8/H12/38). We thank Dr Paul Hayden, of the EnFlo laboratory, University of Surrey, who provided the wind tunnel experimental data and with whom we had helpful discussions. The computations were performed on the Iridis computational system, University of Southampton.

\section{References}

Arnold S., et al.(2004) Introduction to the DAPPLE Air Pollution Project, Sci Total Environ. 332, 139-153.

Baik J.-J., Kang Y.-S., Kim J.-J.(2007) Modeling reactive pollutant dispersion in an urban street canyon, Atmos. Environ. 41, 934-949.

Baker J., Walker H.L., Cai X.M. (2004) A study of the dispersion and transport of reactive pollutants in and above street canyonsa large eddy simulation, Atmos. Environ. 38, 6883-6892.

Britter R.E. and Hanna S.R. (2003) Flow and dispersion in urban areas, Ann. Rev. Fluid Mech. 35 469-496.

Carpentieri M., Robins A.G., Baldi S.(2008) Three-dimensional mapping of wind flow at an urban canyon intersection, to be submitted to J. Wind Eng. Ind. Aerodyn.

Castro I.P., Cheng H. and Reynolds R.(2006) Turbulence over urban-type roughness:decutions from with tunnel measurements, Bound.-Layer Meteoro., 118: 109-131.

Cheng H. and Castro I.P. (2002a) Near wall flow over urban-like roughness, Bound.-Layer Meteorol. 104 229-259.

Cheng H. and Robins A.G. (2004) Wind tunnel simulation of field tracer release in London, In Zhuang F.G. \& Li J.C.(eds) Recent Advances in Fluid Mechanics, Tsinghua University Press \& Springer-Verlag 801-804.

Coceal O., Thomas T.G., Castro I.P. and Belcher S.E. (2006) Mean flow and turbulence statistics over groups of urban-like cubical obstacles, Bound.Layer Meteorol., 121, 491-519.

Dobre A., Arnold S.J., Smalley R.J., Boddy J.W.D., Barlow J.F., Tomlin A.S. and Belcher S.E. (2005) Flow field measurements in the proximity of an urban intersection in London, UK. Atmos. Environ. 39, 4647-4657.

Hamilton J.(2007) Urban air dispersion - modelling fixed and moving sources, MEng thesis, University of Surrey.

Hanna S.R., Tehranian S., Carissimo B., Macdonald R.W. \& Lohner R. (2002) 
Comparisons of model simulations with observations of mean flow and turbulence within simple obstacle arrays, Atmos. Environ. 36 5067-5079.

Kanda M., Moriwaki R. \& Kasamatsu F. (2004) Large-eddy simulation of turbulent organized structures within and above explicitly resolved cube arrays. Bound.-Layer Meteorol. 112 343-368.

Meinders E.R. \& Hanjalić K.(1999) Vortex structure and heat transfer in turbulent flow over a wall-mounted matrix of cubes, Int. J. Heat Fluid Flow 20 255-267.

Moeng C.-H. (1984) A large-eddy simulation model for the study of planetary boundary layer turbulence, J. Atmos. Sci. 41, 2052C2062.

Peric M (2004) Flow simulation using control volumes of arbitrary polyhedral shape, ERCOFTAC Bulletin 62 25-29.

Padhra A., Coceal O. and Barlow J. (2008) Wind profile in urban areas: the influence of building distribution and wind direction. Proc. 8th UK Conference on Wind Engineering, Guildford, 14th-16th, 2008.

Rotach M.W., Gryning S.-E., Batchvarova E., Christen A. and Vogt R. (2004) Pollutant dispersion close to an urban surface - the BUBBLE tracer experiment, Meteorol. Atm. Phys. 87, 39-56.

Shah K.B. (1998) Large eddy simulations of flow past a cubic obstacle. Dissertation. Stanford University.

Smolarkiewicz P.K., Sharman R., Weil J., Perry S.G. c, Heist D., and Bowker G. (2007) Building resolving large-eddy simulations and comparison with wind tunnel experiments, J. Comp. Physics, 227 (1), 633-653.

Stoesser T., Mathey F., Frohlich J. and Rodi W. (2003) LES of flow over multiple cubes. ERCOFTAC Bulletin 56.

Tseng Y.-H., Meneveau C. and Parlange M.B. (2006) Modeling flow around bluff bodies and predicting urban dispersion using large eddy simulation, Environ. Sci. Technol. 40 (8) 2653-2662.

Xie Z.-T., Castro I.P. (2006) LES and RANS for turbulent flow over arrays of wall-mounted cubes, Flow Turbul. Combust., 76 (3), 291-312.

Xie Z.-T., Castro I.P. (2008a) Efficient generation of inflow conditions for large-eddy simulation of street-scale flows, Flow Turbul. Combust., 81(3),449-470.

Xie Z.-T., Castro I.P. (2008b) LES for street-scale environment and its prospects, in: Eds Meyers J., etc, Quality and Reliability of Large-Eddy Simulations, Springer, ERCOFTAC Series, 12, 271-282.

Xie Z.-T., Coceal O. and Castro I.P. (2008c) Large-eddy simulation of flows over random urban-like obstacles, Bound.-Layer Meteorol., 129, 1-23.

Yee E. \& Biltoft C.A. (2004) Concentration fluctuation measurements in a plume dispersing through a regular array of obstacles, Bound.-Layer Meteorol. 111, 363-415.

Yoshizawa A. (1986) Statistical theory for compressible turbulent shear ows, with the application to subgrid scale modelling, Phys. Fluids 29, 2152-2164. 